REGARDS

SUR L'ECONOMIE ALLEMAND

BULLETIN ECONOMIQUE DU CIRAC
Regards sur l'économie allemande

Bulletin économique du CIRAC

$76 \mid 2006$

Varia

\title{
Une croissance propice aux réformes
}

Isabelle Bourgeois

\section{OpenEdition}

Journals

Édition électronique

URL : http://journals.openedition.org/rea/804

DOI : 10.4000/rea. 804

ISBN : 978-2-8218-0848-5

ISSN : 1965-0787

Éditeur

CIRAC

Édition imprimée

Date de publication : 1 mai 2006

Pagination : 3-4

ISSN : 1156-8992

Référence électronique

Isabelle Bourgeois, "Une croissance propice aux réformes », Regards sur l'économie allemande [En ligne], 76 | mai 2006, mis en ligne le 19 juin 2008, consulté le 15 septembre 2020. URL : http:// journals.openedition.org/rea/804 


\section{Une croissance propice aux réformes}

\begin{abstract}
"En ce printemps 2006, l'économie allemande connaît une solide reprise », affirment les instituts de conjoncture dans leur rapport de printemps, présenté le 27 avril. L'embellie qui s'esquissait l'an dernier s'est largement confirmée, et l'évolution de la plupart des indicateurs les amène à revoir nettement à la hausse les prévisions de croissance pour l'année en cours : alors qu'ils ne tablaient que sur 1,2\% dans leur rapport d'automne (voir REA 74/05), ils attendent désormais un taux de 1,8\%. Si leurs prévisions se vérifient, 2006 serait alors la meilleure année depuis 2000 .
\end{abstract}

Car pour la première fois depuis longtemps, la croissance allemande repose sur trois moteurs : les exportations, bien sûr, tirées par un contexte mondial porteur ; les investissements, qui enregistrent une tendance à la hausse depuis 2004 et sont dopés par des carnets de commande bien remplis qui font tourner à haut régime la production industrielle : après avoir rationalisé et réduit leurs surcapacités, les entreprises modernisent aujourd'hui leurs installations et équipements dans un contexte boursier, financier et fiscal favorable. Les forces de croissance externes ont fini par entraîner le troisième moteur, à la grande surprise des instituts : la consommation des ménages s'accroît depuis la fin de l'an dernier et devrait enregistrer une hausse de 0,4\% cette année. Les ménages retrouvent en effet le moral face à un marché de l'emploi moins tendu ; mais ils anticipent aussi l'achat de biens de consommation durable avant que la TVA ne passe à $19 \%$ au $1^{\text {er }}$ janvier 2007, ce qui alimentera le taux de croissance de 0,2 points.

Enfin, l'embellie conjoncturelle commence à se répercuter sur la marché du travail où, depuis le second semestre 2005, le nombre des actifs occupant un emploi soumis à cotisations sociales ne baisse plus. Et si le nombre de chômeurs est en recul, cela traduit bien sûr l'effet des mesures engagées par les réformes Hartz, mais aussi une tendance à la stabilisation, voire à une légère hausse de l'emploi. Elle s'esquisse depuis l'été 2005 : l'industrie ne comprime presque plus ses effectifs et le secteur des services (services aux entreprises, transports et communications, surtout) s'est remis à embaucher.

Les perspectives 2007 sont moins bonnes, la conjoncture faiblira nettement, et le taux de croissance ne devrait plus être que de 1,2 \% sous la conjonction d'effets externes et internes. D'une part, la BCE devrait procéder à une nouvelle hausse de ses taux d'ici la fin 2006, et la conjoncture mondiale se ralentira légèrement. S'y ajoute la grande incertitude quant à l'évolution des prix pétroliers. D'autre part, le gouvernement allemand mènera une politique budgétaire et fiscale restrictive dans l'objectif de réduire le déficit. L'augmentation de 3 points de la TVA se traduira par une hausse du taux d'inflation (de $1,35 \%$ ) si les entreprises la répercutent en totalité sur les prix aux consommateurs. Conjuguée à d'autres hausses des prélèvements fiscaux (tabac, assurances notamment) et sociaux (retraites, santé), elle affectera le pouvoir d'achat des ménages, annulant le récent essor de la consommation. Au total, la croissance sera de ce fait inférieure d'un demi point à ce qu'elle devrait être étant donné le cycle ascendant où se trouve la conjoncture allemande, conclut la majorité des instituts.

L'institut ifo ne partage pas cette analyse, estimant au contraire que, conscients de la dérive budgétaire, les ménages ont anticipé depuis longtemps une hausse des prélèvements, comme en atteste un taux d'épargne qui se maintient à haut niveau et la faiblesse chronique de la consommation. Cette hausse grèvera certes le revenu courant mais non pas le revenu global des ménages, ce qui ne devrait pas restreindre leur consumérisme. L'institut ifo prévoit donc un taux de croissance de 1,7\% pour 2007, une estimation fondée par ailleurs sur " la dynamique conjoncturelle endogène de l'économie allemande ». Car celle-ci a amorcé en 2004 un cycle ascendant ; or ces cycles durent en général quatre ans. L'excellente tenue des exportations, la forte dynamique de l'investissement (biens d'équipement et BTP, ce dernier secteur semblant enfin se normaliser) et la hausse du volume des heures travaillées devraient contrebalancer d'éventuels effets négatifs induits par le poids des prélèvements.

Le gouvernement fédéral estime que ces prévisions sont toutes trop optimistes. II a présenté les siennes le lendemain de la publication du rapport de printemps : elles sont respectivement de $1,6 \%$ et $1,0 \%$ pour 2006 et 2007 . Mais il semblerait que cette prudence ait surtout pour fonction se soustraire aux tentations une 'cagnotte' fiscale plus conséquente que prévu. Alors que le ministre fédéral de l'Economie, Michael Glos (CSU) partage l'analyse des instituts et se réjouit de leur optimisme, son homologue des Finances, Peer Steinbrück (SPD), fait preuve d'une rare réserve. II s'insurge en effet contre la critique qu'ils formulent à l'encontre de la politique projetée par le gouvernement pour
$1,8 \%$ de croissance en 2006 selon les instituts

Trois moteurs de croissance : exportations, investissement et consommation

Stabilisation de l'emploi

2007 : les instituts s'attendent à un tassement conjoncturel..

... sauf l'institut ifo

Le gouvernement fédéral se montre moins optimiste 
réduire le déficit. Non seulement ils estiment que "les hausses fiscales ne mènent à rien car l'expérience enseigne qu'elles nuisent à la croissance et à l'emploi », préférant de loin une réduction des dépenses (subventions, politique pour l'emploi) et leur réorientation de la consommation vers l'investissement, mais ils s'en prennent à un cap de consolidation considéré comme "peu exigeant ».

Prévisions du rapport de printemps 2006 des instituts de conjoncture

\begin{tabular}{|c|c|c|c|c|}
\hline & 2004 & 2005 & 2006 & 2007 \\
\hline \multicolumn{5}{|c|}{ (variation en \% par rapport à la même période de l'année précédente) } \\
\hline PIB & 1,6 & 0,9 & 1,8 & 1,2 \\
\hline Consommation privée & 0,6 & 0,0 & 0,4 & $-0,1$ \\
\hline Consommation publique & $-1,6$ & 0,1 & 0,4 & 0,6 \\
\hline Investisssements bruts en biens d'équipt. & $-0,2$ & $-0,2$ & 3,1 & 3,0 \\
\hline Demande intérieure & 0,6 & 0,3 & 1,2 & 0,7 \\
\hline Exportations & 9,3 & 6,3 & 8,2 & 6,6 \\
\hline Importations & 7,0 & 5,3 & 7,4 & 6,2 \\
\hline Prix à la consommation (2000 = indice 100) & 1,6 & 2,0 & 1,6 & 2,5 \\
\hline Coûts salariaux unitaires & $-1,0$ & $-0,9$ & $-0,7$ & 0,1 \\
\hline \multicolumn{5}{|c|}{ (état) } \\
\hline Déficit public en \% du PIB & $-3,7$ & $-3,3$ & $-2,9$ & $-2,0$ \\
\hline Dette publique (en milliards $€$ ) & $-81,2$ & $-74,5$ & $-67,4$ & $-46,5$ \\
\hline Actifs (en millions) & 38,78 & 38,72 & 38,76 & 38,94 \\
\hline Chômeurs (en millions) & 4,38 & 4,86 & 4,58 & 4,44 \\
\hline
\end{tabular}

Source des données : Rapport de printemps des six instituts de conjoncture, en date du 27-04-2006. NB : les prévisions 2006 et 2007 prennent pour hypothèse un prix moyen du baril de Brent de respectivement $65 \$$ et $60 \$$, un taux de $1,20 \$=1 €$, et une croissance du commerce mondial de $8,5 \%$ puis $7,5 \%$.

Les instituts plaident pour une baisse du poids des prélèvements...

... et mettent en garde contre la tentation d'un 'second tour'
Si les instituts épinglent cette sorte "d'attentisme programmé » (voir REA 75/06) que semble générer la grande coalition, c'est dans une approche constructive de dialogue. Et s'ils ne formulent pas de préconisations (ce rôle ne leur revient pas), ils plaident néanmoins, à défaut d'un retrait de la mesure, pour un échelonnement de la hausse de la TVA (2 points en 2006, puis un autre en 2007), ce qui permettrait de contrefinancer une réforme de la fiscalité des entreprises dont l'objectif premier devrait être de baisser le poids des prélèvements. A l'adresse du gouvernement, ils résument les trois options de réforme en présence, rappelant qu'elles impliquent toutes un changement de paradigme, le droit fiscal actuel n'étant plus révisable, même à la marge.

A l'adresse des partenaires sociaux, ils prônent la poursuite d'une longue politique de modération salariale ; elle a notoirement amélioré la compétitivité-prix de la production et permis de stabiliser l'emploi ; elle devrait se traduire désormais par une tendance à la hausse de l'emploi soumis à cotisations sociales si elle se double d'une hausse de la durée du temps de travail permettant, elle, de comprimer les coûts salariaux. Et ils mettent en garde contre un 'second tour' : une trop forte augmentation des salaires pour absorber le surcoût de l'énergie ou la hausse des prélèvements mettrait à mal l'emploi, accroîtrait l'inflation dans la zone euro et forcerait la BCE à rehausser ses taux en conséquence. L'accord conclu en avril dans la métallurgie constitue à cet égard le maximum possible, et seulement parce que la hausse salariale (3\%) est compensée par une clause de flexibilité respectant les impératifs de compétitivité des entreprises.

Plus généralement, Les instituts JUgent que " dans la politique budgétaire, les intentions affichées et l'action entreprise se contredisent au plus haut degré et ne permettent pas de déceler un quelconque projet à moyen terme, surtout en ce qui concerne le poids futur des prélèvements ". Or cette faible lisibilité risque de déstabiliser la reprise, produisant ainsi l'effet inverse de ce que recherche le gouvernement fédéral en laissant agir les forces de croissance cette année. Et ils le mettent en garde : les périodes de reprise conjoncturelle ne peuvent servir de prétexte pour ajourner les réformes structurelles d'envergure. II ne s'agit plus en effet aujourd'hui de " procéder à de menues corrections laissant les systèmes en l'état, comme ce fut régulièrement le cas par le passé ॥. II y a urgence à mettre en chantier ces réformes de fond permettant enfin de libérer les forces de croissance et de générer de l'emploi. Ils reconnaissent que c'est là une tâche difficile, puisque les divers chantiers (protection sociale, droit du travail, fiscalité, politique familiale) sont interdépendants. Et s'ils ne peuvent être engagés tous en même temps, il faudrait au moins une vision et un affichage clairs des priorités. Au préalable, et condition sine qua non, il s'agit de trancher la question du choix entre plus d'Etat ou plus de marché et de responsabilité individuelle. La grande coalition doit surmonter ses contradictions internes et affronter enfin l'heure des choix. Le contexte conjoncturel est favorable. 